\title{
Human Papillomavirus Serologic Profiles of Selected Filipinos with Head and Neck Squamous Cell Carcinoma
}

\author{
Pia Marie Albano ${ }^{1,2,3}$, Christianne Salvador ${ }^{4}$, Jose Orosa, $\mathrm{III}^{4}$, Sheryl Racelis ${ }^{5}$, Modesty Leaño ${ }^{5}$, Angelika Michel ${ }^{1}$, \\ John Donnie Ramos ${ }^{2,3}$, Dana Holzinger ${ }^{1}$, Michael Pawlita ${ }^{1}$ \\ ${ }^{1}$ Division of Molecular Diagnostics of Oncogenic Infections, Research Program Infection and Cancer, German Cancer Research Center (DKFZ), Heidelberg, Germany; \\ ${ }^{2}$ Department of Biology, College of Science, University of Santo Tomas, Manila; ${ }^{3}$ Research Center for the Natural and Applied Sciences, University of Santo Tomas, Manila; \\ Departments of ${ }^{4}$ ENT Head and Neck Surgery and ${ }^{5}$ Pathology and Laboratories, Mariano Marcos Memorial Hospital and Medical Center, llocos Norte, Philippines
}

\begin{abstract}
Background: The low prevalence of human papillomavirus (HPV) DNA and mRNA in biopsy samples of Filipinos with head and neck squamous cell carcinoma (HNSCC) has been reported previously. Here, the HPV serologic profiles of HNSCC cases were analyzed and associated with lifestyle and sexual practices. Methods: Serum samples were collected between May 2012 and September 2013 from HNSCC patients $(n=22)$ in the northwest region of the Philippines, and age- and sex-matched clinically healthy controls. Antibodies to capsid and early oncoproteins of HPV16, 18, 31, 33, 45, 52, 58, 6, and 11 were analyzed using multiplex serology. Results: Most of the cases were males with tumors of the oral cavity or larynx. Two of the cases tested positive for at least one of the early oncoproteins (E6, $\mathrm{E7}, \mathrm{E} 1$, and/or E2) of HPV16, and 11 did not display reactivity to any HPV early or late oncoproteins. Of the controls, four tested positive for at least one of the HPV16 early oncoproteins, and 10 were non-reactive to all HPV types. Titers to HPV16 E6 or E7 of the seropositive cases and controls were considerably lower than those typically observed in economically developed countries. Conclusions: The low HPV titers seen here are consistent with the results of molecular analyses for this population. Hence, the seropositivity of some of the HNSCC cases is likely an indication of prior exposure to the virus and not the presence of HPV-driven tumors.
\end{abstract}

Key Words: HPV antibodies; Head and neck squamous cell carcinoma; Philippines; Multiplex serology

Received: January 31, 2019 Revised: April 21, 2019 Accepted: May 12, 2019

Corresponding Author: Pia Marie Albano, PhD, Research Center for the Natural and Applied Sciences (RCNAS), Rm. 708 Central Laboratories, University of Santo Tomas, España Blvd, Manila, Philippines

Tel: +63-2-4061661 (loc 8545), Fax: +63-2-7314031, E-mail: psalbano@ust.edu.ph, p.albano2013@gmail.com

Human papillomavirus (HPV) has been demonstrated in more than $99 \%$ of cervical cancers and over $90 \%$ of their squamous intra-epithelial precursor lesions. ${ }^{1}$ Moreover, molecular and epidemiologic studies have demonstrated that HPV, particularly HPV16, also plays a role in the pathogenesis of a subset of head and neck squamous cell carcinomas (HNSCC) particularly oropharyngeal squamous cell carcinoma (OPSCC). ${ }^{2,3}$ Biomarkers of HPV-driven cancers include DNA and mRNA detected in tumors using in situ hybridization and polymerase chain reaction-based assays, expressed cellular proteins (such as pRb, p 53 , and $\mathrm{p} 16^{\mathrm{INK} 4 \mathrm{a}}$ ) measured using immunohistochemistry, and serological markers indicative of cumulative viral exposure.

There is strong correlation between HNSCC and seropositivity to the E6 and E7 oncoproteins of HPV16. ${ }^{4-7}$ The risk of HPVdriven HNSCC is much greater in individuals positive for anti- bodies to HPV16 E6 or E7 than in those positive for antibodies to HPV16 virus-like particles. Seropositivity to HPV18 E6, HPV33 E6, and HPV58 E7 are strongly associated with OPSCC and HPV52 E7 with oral cavity squamous cell carcinoma (OSCC). ${ }^{8}$ Due to the low prevalence of HPV16 E6 or E7 antibodies in healthy individuals without an HPV-related tumor, HPV antibodies are now being considered for monitoring for previous exposure, measuring prognosis, and monitoring treatment ${ }^{9-13}$ of HPV-associated cancers. ${ }^{14}$

The low prevalence of HPV DNA and mRNA among HNSCC cases from the northwest region of the Philippines was reported previously. ${ }^{15}$ In the current study, serum samples of HNSCC patients from the same region along with their age- and sexmatched clinically healthy controls were analyzed for antibodies to the capsid protein (L1), early oncoproteins (E6, E7), and other 
early proteins (E1, E2) of the carcinogenic mucosal HPV16, 18, $31,33,45,52$, and 58 genotypes and the non-carcinogenic mucosal HPV6 and 11 genotypes to determine whether frequency of seropositivity and antibody titers are in concordance with molecular analyses. Moreover, the results of serologic analyses were correlated with lifestyle risk factors including sexual practices.

\section{MATERIALS AND METHODS}

\section{Sample population and study site}

The Mariano Marcos Memorial Hospital and Medical Center (MMMH-MC) in Ilocos Norte, Philippines, served as the study base. The study included cases of newly diagnosed, histologically confirmed primary tumors of the oral cavity (OSCC), oropharynx (OPSCC), or larynx (laryngeal squamous cell carcinoma [LSCC]) seen at MMMH-MC between May 2012 and September 2013. The study also required that the cases had no prior history of cervical dysplasia and/or cervical cancer. The cases were age- $( \pm$ 2 years) and sex-matched with volunteer cancer-free controls recruited from the same communities where the study cases resided.

All participants completed a standardized questionnaire, either through self-administration or an interview with a member of the research group, that determined their alcohol consumption, tobacco use, and sexual practices. The clinical data of the patients were retrieved from medical records and histopathological reports. Blood was collected from both patients and controls at the time of the interview. The serum was separated and stored immediately at $-80^{\circ} \mathrm{C}$ until use.

It must be noted that the freshly frozen biopsy samples of the HNSCC cases at this institution were previously analyzed for HPV DNA and mRNA. ${ }^{15}$ Only one of the cases tested positive for HPV DNA and RNA specifically to the low-risk HPV $11 .{ }^{15}$

\section{Multiplex serology}

All serum samples were subjected to multiplex serology, a high-throughput technology that allowed the simultaneous quantification of specific antibodies against the major capsid protein (L1), the early oncoproteins (E6, E7), and other early proteins (E1, E2) of the carcinogenic mucosal HPV16 and HPV18 genotypes (L1, E1, E2, E6, and E7), the carcinogenic mucosal HPV31, HPV33, HPV45, HPV52, and HPV58 genotypes (E6, E7, and L1), and the non-carcinogenic mucosal HPV6 and HPV11 genotypes (E6, E7, and L1). The assay makes use of viral antigens bacterially expressed as glutathione
S-transferase (GST) fusion proteins. ${ }^{16,17}$ Spectrally distinct bead sets (SeroMAP Microspheres, Luminex Corp., Austin, TX, USA) carrying different viral antigens were individually washed and subsequently mixed. Each serum sample was diluted (1:100) in pre-incubation buffer (phosphate buffered saline with ChemiBlock), combined with the mixed beads, and incubated. The bound antibodies were detected with biotinylated anti-human secondary antibodies (goat anti-human $\operatorname{IgG}[\mathrm{H}+\mathrm{L}], \operatorname{IgA}$, and IgM) (Dianova, Hamburg, Germany) followed by conjugate streptavidin-R-phycoerythrin. With a Luminex 200 analyzer (Luminex Corp.), the reporter fluorescence of the beads was measured and expressed as the mean fluorescence intensity (MFI) of at least 100 beads per set per serum sample. For background determination, beads were loaded with GST alone. Net MFI values were categorized as either antibody positive (reactive) or negative (nonreactive) based on cut-off values of the mean $\pm 3 \mathrm{SD}$ (standard deviation) excluding the positive outliers of the MFI values of 25 sera from self-declared sexually-naïve Indian girls aged $12-15$ years old. ${ }^{4,18}$ Additionally, the minimal cut-off was set to 300 MFI for early HPV antigens and 400 MFI for L1 antigens.

\section{Data analysis}

IBM Statistical Package for Social Sciences (IBM SPSS, IBM Corp., Armonk, NY, USA) was used in the analysis of the data. The chi-square test of homogeneity was used to compare nominal data, and Fisher exact test was used to compare expected frequencies less than 5.00 (i.e., number of lifetime sex partners, performs oral-genital sex, engages in casual and/or commercial sex, engages in same-sex sexual activity, sex partner with history of sexually transmitted infection, and personal history of sexuallytransmitted disease). The Mann-Whitney rank-sum test was used to compare median scores.

\section{Ethics statement}

The design, sampling, experimental protocols, questionnaires, and other pertinent documents were reviewed and approved by the Research Ethics Review Committee (RERC) of MMMHMC. All participants gave their written informed consent.

\section{RESULTS}

This study considered a total of 22 HNSCC cases (13 OSCC, 2 OPSCC, and 7 LSCC) during the 16-month recruitment period. All HNSCC patients that visited MMMH-MC were invited to participate, but some of those diagnosed with late-stage cancer or of extreme age declined to participate. Moreover, two of the 
cases were also not paired since it was difficult to recruit controls that matched their age ( $\geq 80$ years old). There were more males $(n=13)$ than females, and the median age at initial diagnosis was 64 years old (range, 41 to 87 years). A majority of the cases had well-differentiated tumors $(n=18)$ and presented with the late stage of the disease $(n=17)$.

There were no significant differences between the cases and controls in terms of the number of lifetime sex partners $(p>.99)$ and engagement in oral sex $(\mathrm{p}=.332)$. Although more controls engaged in casual or commercial sex $(\mathrm{p}=.018)$, more controls also used condoms for protection $(\mathrm{p}=.029)$. Very few of the participants, whether case or control, had more than five lifetime sex partners or engaged in oral sex (Table 1).

The HPV serologic profiles of the cases were the same from diagnosis to treatment. Among the 22 cases, only two (9\%, both OSCC) showed reactivity to the E6 protein: one case to both HPV18 and HPV 45 and one to HPV31. Four (18\%, 2 LSCC and 2 OSCC) were reactive to the $\mathrm{E} 7$ protein: two cases to HPV33, one to HPV16, and one to HPV18. None of the cases showed dual reactivity to E6 and E7 of the same HPV genotype. One OSCC case was reactive to $\mathrm{E} 2$ of HPV18 and another was reactive to E1 of HPV18 and E2 of HV16 (Table 2). Seven (31.8\%, 4 OSCC and 3 LSCC) showed multiple reactivity to the L1 protein of HPV6, 11, 16, 18, 31, 33, 45, 52, and/or 58 (Table 2). Further, two of the LSCC cases were positive to HPV 11. Overall, only two $(9 \%)$ of the cases were positive to at least one of the early proteins (E6, E7, E1, and/or E2) of HPV16, and 11 (50\%) did not display reactivity to any of the early or late proteins of HPV.

Only three (15\%) among the controls showed reactivity to either E6 or E7: one to the E6 protein of both HPV11 and HPV52 and two to E7 of either HPV16 or HPV33. One of the controls showed dual reactivity to E1 and E2 of both HPV16 and HPV18. Another control was reactive only to E2 of HPV16 (Table 3). Seven of the controls (35\%) displayed multiple reactivity to L1 of HPV6, 11, 16, 18, 31, 33, 45, 52, and/or 58 (Table 3). Overall, 10 of the controls $(50 \%)$ were not reactive to any of the HPV proteins, and four $(20 \%)$ were positive to at least one of the HVP16 early proteins.

\section{DISCUSSION}

In this study, seropositivity to the early and late proteins of HPV was found in only half of the HNSCC cases and clinically healthy controls. None of the participants showed dual reactivity to the E6 and E7 proteins of HPV16, which is the most associated with HNSCC, particularly OPSCC. ${ }^{5,8,14,19}$ Increased titers of
Table 1. Clinico-epidemiologic profile of the cases vs controls $(n=42)$

\begin{tabular}{|c|c|c|c|}
\hline Characteristic & $\begin{array}{l}\text { Case } \\
(n=22)\end{array}$ & $\begin{array}{l}\text { Control } \\
(n=20)\end{array}$ & $\begin{array}{c}p- \\
\text { value }^{a}\end{array}$ \\
\hline Age (yr) & $63(41-87)$ & $62(39-74)$ & \\
\hline \multicolumn{4}{|l|}{ Sex } \\
\hline Male & $13(59)$ & $11(55)$ & \\
\hline Female & $9(41)$ & $9(45)$ & \\
\hline \multicolumn{4}{|l|}{ Tumor site } \\
\hline Larynx & 7 (32) & - & \\
\hline Oral cavity & $13(59)$ & - & \\
\hline Oropharynx & $2(9)$ & - & \\
\hline \multicolumn{4}{|l|}{ Tumor grade } \\
\hline Well-differentiated & $18(82)$ & - & \\
\hline Moderately-differentiated & $3(14)$ & - & \\
\hline Poorly-differentiated & $1(4)$ & - & \\
\hline \multicolumn{4}{|l|}{ Tumor category } \\
\hline Tis, T1, T2 & $5(23)$ & - & \\
\hline $\mathrm{T} 3, \mathrm{~T} 4$ & $17(77)$ & - & \\
\hline Alcohol consumption & & & .516 \\
\hline Never & $11(50)$ & $8(40)$ & \\
\hline Ever & $11(50)$ & $12(60)$ & \\
\hline Tobacco use & & & .052 \\
\hline Never & $3(14)$ & $8(40)$ & \\
\hline Ever & $19(86)$ & $12(60)$ & \\
\hline Median age at first sexual intercourse (yr) & $\begin{array}{c}20.5 \\
(19-24)\end{array}$ & $\begin{array}{c}21 \\
(17.5-25)\end{array}$ & .870 \\
\hline No. of lifetime sex partners & & & $>.99$ \\
\hline $0-5$ & $19(86)$ & $17(85)$ & \\
\hline$\geq 6$ & $3(14)$ & $3(15)$ & \\
\hline Performs oral-genital sex & & & .332 \\
\hline Yes & $3(14)$ & $5(25)$ & \\
\hline No & $19(86)$ & $14(70)$ & \\
\hline No answer & 0 & $1(5)$ & \\
\hline Engages in casual and/or commercial sex & & & .018 \\
\hline Yes & 0 & $5(25)$ & \\
\hline No & $22(100)$ & $15(75)$ & \\
\hline Engages in same-sex sexual activity & & & .099 \\
\hline Yes & 0 & $3(15)$ & \\
\hline No & $22(100)$ & $17(85)$ & \\
\hline Sex partner has history of STD & & & .003 \\
\hline Yes & 0 & $1(5)$ & \\
\hline No & $22(100)$ & $13(65)$ & \\
\hline Uncertain/no answer & 0 & $6(30)$ & \\
\hline Personal history of STD & & & .221 \\
\hline Yes & 0 & $2(10)$ & \\
\hline No & $22(100)$ & $18(90)$ & \\
\hline Use of condom & & & .029 \\
\hline Yes & $6(27)$ & $13(65)$ & \\
\hline No & $16(73)$ & $7(35)$ & \\
\hline
\end{tabular}

Values are presented as median (range) or number (\%).

aChi-square test of homogeneity was used to compare nominal data while Fisher's exact test was used to compare expected frequencies less than 5.00 (i.e., number of lifetime sex partners, performs oral-genital sex, engages in casual and/or commercial sex, engages in same-sex sexual activity, sex partner with history of sexually-transmitted disease [STD], and personal history of STD). Mann-Whitney rank-sum test was used to compare median scores. 
Table 2. HPV serologic profile of the cases to the early and late proteins of low-risk (HPV6, 11) and high-risk (HPV16, 18, 31, 33, 45, 52, and 58) types

\begin{tabular}{|c|c|c|c|c|c|c|c|}
\hline \multirow{2}{*}{ Case ID } & \multirow{2}{*}{ Sex } & \multirow{2}{*}{$\begin{array}{l}\text { Age at initial } \\
\text { diagnosis (yr) }\end{array}$} & \multirow{2}{*}{ Tumor site } & \multirow{2}{*}{ HPV DNA } & \multirow{2}{*}{ HPV mRNA } & \multicolumn{2}{|c|}{ Seropositivity } \\
\hline & & & & & & Early proteins (E6, E7, E1, E2) & Late proteins (L1) \\
\hline $18-1$ & $\mathrm{M}$ & 49 & LSCC & HPV11 L1 ${ }^{b}$ & HPV11 E6 flb & Negative & HPV11 ${ }^{b}$ \\
\hline $09-1$ & $\mathrm{~F}$ & 59 & Oscc & Negative & Negative & HPV18 E6, HPV45 E6 ${ }^{b}$ & HPV16, 31, 33, 52, 58 b \\
\hline $11-1$ & $\mathrm{~F}$ & 65 & Oscc & Negative & Negative & HPV31 E6 $6^{b}$ & HPV $31^{b}$ \\
\hline $22-1$ & M & 78 & oscc & Negative & Negative & HPV18 E1, HPV16 E2 ${ }^{b}$ & HPV11, 18, 31, 33, 45, 52, 58b \\
\hline $07-1$ & $\mathrm{~F}$ & 53 & Oscc & Negative & Negative & HPV33 E7 $7^{b}$ & Negative \\
\hline $14-1$ & $M$ & 59 & LSCC & Negative & Negative & HPV33 E7 ${ }^{b}$ & Negative \\
\hline $17-1$ & $M$ & 41 & LSCC & Negative & Negative & HPV16 E7 ${ }^{b}$ & Negative \\
\hline $21-1$ & $\mathrm{~F}$ & 69 & Oscc & Negative & Negative & HPV18 E7, HPV18 E2 ${ }^{\mathrm{b}}$ & Negative \\
\hline $06-1$ & $\mathrm{~F}$ & 70 & Oscc & Negative & Negative & Negative & HPV58 ${ }^{\mathrm{b}}$ \\
\hline $15-1$ & $M$ & 59 & LSCC & Negative & Negative & Negative & HPV33 ${ }^{b}$ \\
\hline $19-3$ & $M$ & 69 & LSCC & Negative & Negative & Negative & HPV6, 11, $16^{b}$ \\
\hline $01-1$ & $M$ & 87 & OscC & Negative & Negative & Negative & Negative \\
\hline $02-1$ & $\mathrm{~F}$ & 76 & OPSCC & Negative & Negative & Negative & Negative \\
\hline 03-1 & $M$ & 57 & OPSCC & Negative & Negative & Negative & Negative \\
\hline 04-1 & $M$ & 57 & oscc & Negative & Negative & Negative & Negative \\
\hline $05-1$ & $M$ & 77 & oscc & Negative & Negative & Negative & Negative \\
\hline 08-1 & $\mathrm{F}$ & 51 & oscc & Negative & Negative & Negative & Negative \\
\hline $10-1$ & $\mathrm{~F}$ & 69 & Oscc & Negative & Negative & Negative & Negative \\
\hline $12-1$ & $\mathrm{~F}$ & 70 & OScC & Negative & Negative & Negative & Negative \\
\hline $13-1$ & $M$ & 55 & Oscc & Negative & Negative & Negative & Negative \\
\hline $16-1$ & $M$ & 61 & LSCC & Negative & Negative & Negative & Negative \\
\hline $20-1$ & $M$ & 68 & LSCC & Negative & Negative & Negative & Negative \\
\hline
\end{tabular}

HPV, human papillomavirus; LSCC, laryngeal squamous cell carcinoma; OSCC, oral cavity squamous cell carcinoma; OPSCC, oropharyngeal squamous cell carcinoma.

${ }^{a}$ Molecular analyses done earlier ${ }^{15}$; bPositive for HPV DNA, HPV mRNA, or HPV antibodies.

Table 3. HPV serologic profile of the controls to the early and late proteins of low-risk (HPV6, 11) and high-risk (HPV16, 18, 31, 33, 45, 52, and 58) types

\begin{tabular}{|c|c|c|c|c|}
\hline \multirow{2}{*}{ Control ID } & \multirow{2}{*}{ Sex } & \multirow{2}{*}{ Age (yr) } & \multicolumn{2}{|c|}{ Seropositivity } \\
\hline & & & Early proteins (E6, E7, E1, E2) & Late proteins (L1) \\
\hline $06-0$ & $\mathrm{~F}$ & 71 & HPV16 E1, HPV18 E1, HPV16 E2, HPV18 E2a & HPV11, 18, 31, 33, 45 52, 58 \\
\hline $07-0$ & $\mathrm{~F}$ & 53 & HPV16 E7 ${ }^{\mathrm{a}}$ & HPV11, 16, 18, 31, 45a \\
\hline 03-0 & M & 59 & HPV33 E7, HPV16 E1 a & Negative \\
\hline $12-0$ & $\mathrm{~F}$ & 72 & HPV16 E2 ${ }^{\mathrm{a}}$ & Negative \\
\hline $20-0$ & M & 65 & HPV11 E6, HPV52 E6 ${ }^{a}$ & Negative \\
\hline $08-0$ & $\mathrm{~F}$ & 54 & Negative & HPV6, 11, 58 ${ }^{\mathrm{a}}$ \\
\hline $14-0$ & M & 59 & Negative & HPV11, 16, 31, 33, 45, 52, 58a \\
\hline $15-0$ & M & 61 & Negative & HPV11 ${ }^{\mathrm{a}}$ \\
\hline $17-0$ & M & 39 & Negative & HPV11, $31^{a}$ \\
\hline $18-0$ & $M$ & 47 & Negative & HPV $18,58^{a}$ \\
\hline $02-0$ & $\mathrm{~F}$ & 74 & Negative & Negative \\
\hline $04-0$ & M & 55 & Negative & Negative \\
\hline 05-0 & M & 74 & Negative & Negative \\
\hline $09-0$ & $\mathrm{~F}$ & 58 & Negative & Negative \\
\hline $10-0$ & $\mathrm{~F}$ & 68 & Negative & Negative \\
\hline $11-0$ & $\mathrm{~F}$ & 63 & Negative & Negative \\
\hline $13-0$ & M & 53 & Negative & Negative \\
\hline $16-0$ & $M$ & 64 & Negative & Negative \\
\hline $19-0$ & $M$ & 66 & Negative & Negative \\
\hline $21-0$ & $\mathrm{~F}$ & 66 & Negative & Negative \\
\hline
\end{tabular}

aPositive for human papillomavirus (HPV) antibodies. 
HPV18 E6, HPV33 E6, and HPV58 E7 antibodies are strongly associated with OPSCC and HPV52 E7 with OSCC. ${ }^{8}$ The two OPSCC cases here were negative to all early and late HPV proteins; however, two of the OSCC cases were positive to HPV 52 L1 but not to E7 nor to any of the early proteins. The seropositive clinically healthy controls were primarily reactive to the early (E1 and E2) and late (L1) proteins. It must also be noted that antibody titers to HPV16 E6 or E7 in both the seropositive cases and controls were considerably lower compared to regions where the prevalence of HPV-driven HNSCC is high. ${ }^{5,8,14,19}$ Thus, the findings here correspond to the results of molecular analyses done earlier on biopsy samples of the cases. ${ }^{15}$

Among the cases, one (case 18-1) tested positive for both the DNA and mRNA of HPV11, a low-risk HPV type commonly associated with recurrent respiratory papillomatosis (RRP). The patient also registered very high titers of antibodies to the late, but not the early proteins of HPV11 (Table 2). It is worth noting that this patient had RRP prior to the diagnosis of LSCC. HPV11 sometimes causes cancer if genetic susceptibility and immune suppression allow the virus to persist with deregulated patterns of gene expression..$^{20}$ The other HPV11 L1 seropositive case (case 19-3) was negative for HPV DNA and mRNA and had no history of RRP, as indicated in his medical records (Table 2).

Antibodies to E6 and E7 of HPV18, 33, and 58 are associated with OPSCC and LSCC ${ }^{8,10,21}$ and HPV52 with a risk of OSCC and OPSCC. ${ }^{5,8}$ The HPV18 or 33 seropositive cases here were either OSCC or LSCC, and none had dual reactivity to at least two early proteins of the same HPV genotype, which has been suggested as a biomarker of HPV-driven HNSCC. ${ }^{5,13}$ Hence, the HPV seropositivity seen here may be a marker of recent HPV exposure.

It has been hypothesized that anti-E1 and E2 antibodies likely develop prior to full transformation, overexpression, and subsequent seroconversion to E6 and E7 because E1 and E2 are expressed at such low levels in HPV-transformed cells. ${ }^{11}$ However, seropositivity to E6 and E7 is usually detectable only after tumor invasion, likely because a humoral response is generated only upon the release and spread of E6 and E7 oncoproteins into systemic circulation, which happens after a tumor vascular bed has been created followed by necrosis. ${ }^{5}$ Dual seropositivity to E6 and any one of the other early viral proteins (E7, E1, or E2) of one HPV type has a sensitivity of $95 \%$ and a specificity of $98 \%$, indicating they could serve as reliable markers for diagnosis and prognosis of HNSCC. ${ }^{10,22,23}$

The cases and controls in this study appeared to be sexually conservative (i.e., a small number of lifetime sex partners, do not perform oral sex) compared to people from more economically developed regions. Thus, there was a lower risk of HPV-driven HNSCC, which is believed to be transmitted through sexual means. ${ }^{24,25}$ The characteristics of the cases here were more consistent with the features of HPV-negative HNSCC; the age at onset of their HNSCC was in the 6th to 7th decade of life, and a majority were tobacco and alcohol users. Dual seropositivity to E6 and $\mathrm{E} 7$ is much more common among young males with a greater number of lifetime sex partners, ${ }^{13}$ an observation that is consistent with the characteristics observed in patients with HPVdriven HNSCC. ${ }^{26}$

This study included a limited number of cases from a single region of the country where HNSCC was most prevalent because there are no nationwide biobanks and participants were only recruited at the time this study was initiated. However, the cases were strictly age- and sex-matched with physician-assessed clinically healthy controls recruited from the same geographical location for comparison of HPV antibody titers.

Serological assays are not usually used in HPV diagnosis due to their low sensitivity and tumor-site specificity. However, HPV serologic markers can be used to assess previous exposure, measure prognosis, and monitor treatment. ${ }^{8,10-13}$ The current study was done to assess HPV exposure of HNSCC cases and their matched controls. The results of the serological analyses, lifestyle risk factors, and sexual practices of the HNSCC cases in this study complement the results of previous molecular analyses for presence of DNA and mRNA in tumors of retrospective HNSCC cases from the same region. ${ }^{15}$ Hence, it can be inferred that the low HPV titers seen among the participants were indicative of prior exposure and not due to presence of HPV-driven tumors.

A follow-up study involving a greater number of cases from both rural and urban areas in the Philippines should be conducted to determine any significant increase in the incidence of HPVrelated HNSCC as verified using molecular and serologic analyses. In the present study, the median age of the cases ( 59 years old) and controls ( 59 years old) who were HPV seropositive was lower compared to the seronegative cases (68 years old) and controls (65 years old), indicating that the younger generations are more liberal. Given the strong influence of social media, Filipinos are becoming more sexually permissive, which increases their risk for HPV-driven HNSCC.

This study adds to the existing evidence that individuals without HPV-related tumors typically have low levels of HPV16 E6 or E7 antibodies. It supports the idea that HPV antibody testing can be used as a cheaper alternative to complex molecular marker 
analyses in screening for HPV-driven cancers especially in countries where resources are limited.

\section{ORCID}

Pia Marie Albano: https://orcid.org/0000-0002-5024-8819

Angelika Michel: https://orcid.org/0000-0001-7684-4742

John Donnie Ramos: https://orcid.org/0000-0001-9433-106X

Dana Holzinger: https://orcid.org/0000-0002-5010-5236

Michael Pawlita: https://orcid.org/0000-0002-4720-8306

\section{Author Contributions}

Conceptualization: PMA, JO, JDR, DH, MP.

Data curation: PMA, CS, SR.

Funding acquisition: PMA, MP.

Investigation: PMA, CS, JO, SR, ML.

Methodology: PMA, AM, DH, MP.

Project administration: PMA, JO, ML, JDR, DH, MP.

Resources: PMA, CS, JO, SR, ML, MP.

Supervision: JO, ML, JDR, DH, MP.

Validation: DH, MP.

Visualization: PMA, JDR, DH, MP.

Writing—original draft: PMA, DH, MP.

Writing—review \& editing: PMA, DH, MP.

\section{Conflicts of Interest}

MP has received royalties for patents owned by DKFZ and research support through cooperate contracts between DKFZ and Roche and Qiagen in the field of HPV diagnostics. However, the funders had no role in study design, data collection and analysis, decision to publish, or preparation of the manuscript.

\section{Funding}

PMA was supported through grants from the Department of Science and Technology (DOST) and the Commission on Higher Education of the Philippines (CHEd).

\section{Acknowledgments}

Our thanks go out to Ute Koch and Monika Oppenlaender for technical assistance.

\section{REFERENCES}

1. Monie A, Hung CF, Roden R, Wu TC. Cervarix: a vaccine for the prevention of HPV 16, 18-associated cervical cancer. Biologics 2008; 2: 97-105.
2. D'Souza G, Dempsey A. The role of HPV in head and neck cancer and review of the HPV vaccine. Prev Med 2011; 53 Suppl 1: S5-11.

3. D'Souza G, Kreimer AR, Viscidi R, et al. Case-control study of human papillomavirus and oropharyngeal cancer. N Engl J Med 2007; 356: 1944-56.

4. Kreimer AR, Pierce Campbell CM, Lin HY, et al. Incidence and clearance of oral human papillomavirus infection in men: the HIM cohort study. Lancet 2013; 382: 877-87.

5. Ribeiro KB, Levi JE, Pawlita M, et al. Low human papillomavirus prevalence in head and neck cancer: results from two large casecontrol studies in high-incidence regions. Int J Epidemiol 2011; 40: 489-502.

6. Wyss A, Hashibe M, Chuang SC, et al. Cigarette, cigar, and pipe smoking and the risk of head and neck cancers: pooled analysis in the International Head and Neck Cancer Epidemiology Consortium. Am J Epidemiol 2013; 178: 679-90.

7. Herrero R, Castellsagué X, Pawlita M, et al. Human papillomavirus and oral cancer: the International Agency for Research on Cancer multicenter study. J Natl Cancer Inst 2003; 95: 1772-83.

8. Michaud DS, Langevin SM, Eliot M, et al. High-risk HPV types and head and neck cancer. Int J Cancer 2014; 135: 1653-61.

9. Lang Kuhs KA, Pawlita M, Gibson SP, et al. Characterization of human papillomavirus antibodies in individuals with head and neck cancer. Cancer Epidemiol 2016; 42: 46-52.

10. Kreimer AR, Johansson M, Waterboer T, et al. Evaluation of human papillomavirus antibodies and risk of subsequent head and neck cancer. J Clin Oncol 2013; 31: 2708-15.

11. Anderson KS, Wong J, D'Souza G, et al. Serum antibodies to the HPV16 proteome as biomarkers for head and neck cancer. Br J Cancer 2011; 104: 1896-905.

12. Rubenstein LM, Smith EM, Pawlita M, Haugen TH, Hamšíková E, Turek LP. Human papillomavirus serologic follow-up response and relationship to survival in head and neck cancer: a case-comparison study. Infect Agent Cancer 2011; 6: 9.

13. Smith EM, Pawlita M, Rubenstein LM, Haugen TH, Hamsikova E, Turek LP. Risk factors and survival by HPV-16 E6 and E7 antibody status in human papillomavirus positive head and neck cancer. Int J Cancer 2010; 127: 111-7.

14. Smith EM, Ritchie JM, Pawlita M, et al. Human papillomavirus seropositivity and risks of head and neck cancer. Int J Cancer 2007; 120: 825-32.

15. Albano PM, Holzinger D, Salvador C, et al. Low prevalence of human papillomavirus in head and neck squamous cell carcinoma in the northwest region of the Philippines. PLoS One 2017; 12: e0172240.

16. Waterboer T, Sehr P, Pawlita M. Suppression of non-specific binding in serological Luminex assays. J Immunol Methods 2006; 309: 
200-4.

17. Waterboer T, Sehr P, Michael KM, et al. Multiplex human papillomavirus serology based on in situ-purified glutathione s-transferase fusion proteins. Clin Chem 2005; 51: 1845-53.

18. Clifford GM, Shin HR, Oh JK, et al. Serologic response to oncogenic human papillomavirus types in male and female university students in Busan, South Korea. Cancer Epidemiol Biomarkers Prev 2007; 16: 1874-9.

19. Anantharaman D, Gheit T, Waterboer T, et al. Human papillomavirus infections and upper aero-digestive tract cancers: the ARCAGE study. J Natl Cancer Inst 2013; 105: 536-45.

20. Egawa N, Doorbar J. The low-risk papillomaviruses. Virus Res 2017; 231: 119-27.

21. Baboci L, Boscolo-Rizzo P, Holzinger D, et al. Evidence of the causal role of human papillomavirus type 58 in an oropharyngeal carci- noma. Virol J 2013; 10: 334.

22. Boscolo-Rizzo P, Pawlita M, Holzinger D. From HPV-positive towards HPV-driven oropharyngeal squamous cell carcinomas. Cancer Treat Rev 2016; 42: 24-9.

23. Broglie MA, Jochum W, Michel A, et al. Evaluation of type-specific antibodies to high risk-human papillomavirus (HPV) proteins in patients with oropharyngeal cancer. Oral Oncol 2017; 70: 43-50.

24. Lajer CB, von Buchwald C. The role of human papillomavirus in head and neck cancer. APMIS 2010; 118: 510-9.

25. Smith EM, Ritchie JM, Summersgill KF, et al. Age, sexual behavior and human papillomavirus infection in oral cavity and oropharyngeal cancers. Int J Cancer 2004; 108: 766-72.

26. Chaturvedi AK. Epidemiology and clinical aspects of HPV in head and neck cancers. Head Neck Pathol 2012; 6 Suppl 1: S16-24. 